\title{
DESIGN AND ANALYSIS OF SPIRAL WHEEL RIM FOR FOUR WHEELER
}

\author{
A. SUGANTHI ${ }^{1}$, M. SIVAPRIYA ${ }^{2}$, T. NARAYANAN ${ }^{3} \&$ DR. R. RAJAPPAN ${ }^{4}$ \\ ${ }^{1,2,3}$ Assistant Professor, Mailam Engineering College, Tamil Nadu, India \\ ${ }^{4}$ Professor, Mailam Engineering College, Tamil Nadu, India
}

\begin{abstract}
Alloy wheels are automobile wheels which are made from an alloy of aluminium or magnesium metals or sometimes a mixture of both. Alloy wheels differ from normal steel wheels because of their lighter weight, which improves the steering and the speed of the car. Alloy wheels will reduce the unstrung weight of a vehicle compared to one fitted with standard steel wheels. The benefit of reduced unstrung weight is more precise steering as well as a nominal reduction in fuel consumption. Alloy is an excellent conductor of heat, improving heat dissipation from the brakes, reducing the risk of brake failure under demanding driving conditions. At present four wheeler wheels are made of Aluminium Alloys. In this project a parametric model is designed for Alloy wheel used in four wheelers by collecting data from reverse engineering process from existing model. Design is evaluated by analysing the model by changing the design of rim styles to be strong and balanced. Its material should not deteriorate with weathering and corrosion.

KEYWORDS: Al Alloy, Mg Alloy, Lighter Weight, Heat Dissipation
\end{abstract}

Received: Jun 06, 2020; Accepted: Jun 26, 2020; Published: Aug 24, 2020; Paper Id.: IJMPERDJUN2020864

\section{INTRODUCTION}

The importance of wheels and tyres in the automobile is obvious. Without the engine the car may be towed but even that is not possible without wheels. The wheel along with tyre has to take the vehicle load, provide a cushioning effect and cope up with the steering control. The various requirements of an automobile wheels are:

- It must be strong enough to perform the above functions.

- It should be balanced both statically as well as dynamically.

- It should be as light as possible so that the unsprung weight is least.

- It should be possible to remove or mount the wheel easily.

- Its material should not deteriorate with weathering and age. In case the material is susceptible to corrosion, it must be given suitable protective treatment.

\section{WHEEL RIMS}

\section{Magnesium Alloy Rims}

Some magnesium rims are weak and unsuitable for heavy off-road operations, for example magnesium fitted to the Mercedes-G. But many are quite strong enough for most uses. Their disadvantage comes only if when they are bent, when they are much more difficult to repair and a hammer and a tree trunk aren't going to bend them straight: It will just break them more. Steel is the better, but heavier and uglier choice. 


\section{Steel Rims}

Steel rims are constructed in two parts: a pressed steel centre boss and a rolled circular bed for the tyre. These parts are either riveted or welded together, riveted types being the strongest and most reliable. Steel rims are sometimes of inferior quality and in some cases severely warped rims are supplied with new vehicles, making perfect balancing impossible.

\section{Split Rims}

Some older vehicles were fitted with split rims of a two part design. This facilitates the removal of the tyre from the rim. It is imperative that the tyre be totally deflated prior to splitting the rim as air pressure remaining in the tyre will cause the protective gaiter consisting of a ring of rim to split with explosive force which could cause serious injury. Also, when a tube is fitted onto a split rim, a shaped rubber must be inserted between the rim and the tube, without which tube will get damage.

\section{OBJECTIVE}

The objective of this project is to design and structural analysis wheel rim for four wheeler according to material change. Two kind of test is performed

- Bending endurance test

- Radial endurance test

- Pressure loading

- Centrifugal loading

- Vertical loading

\section{Theoretical Aspects of a Problem}

\section{The Geometry of the Wheels}

The solid model of the wheel rim is created in catia. This shows the complete solid rim with 9 flectures and 5 bolts. The wheel is modelled according to the standards.

\section{Producing a Mesh}

After creating a model the wheel is transferred to the ANSYS using IGES translator, it is then meshed using auto mesh by parts. Throughout the model 10-noded tetrahedral solid elements are used.

\section{Element Groups and Material Properties Used}

Aluminium alloy is used for the wheel. Appropriate material properties have been used for the elements in these areas. In the case of bending test, three types of element groups are defined, 8-noded tetrahedral solid element 3D elastic beam Right bar Element. 


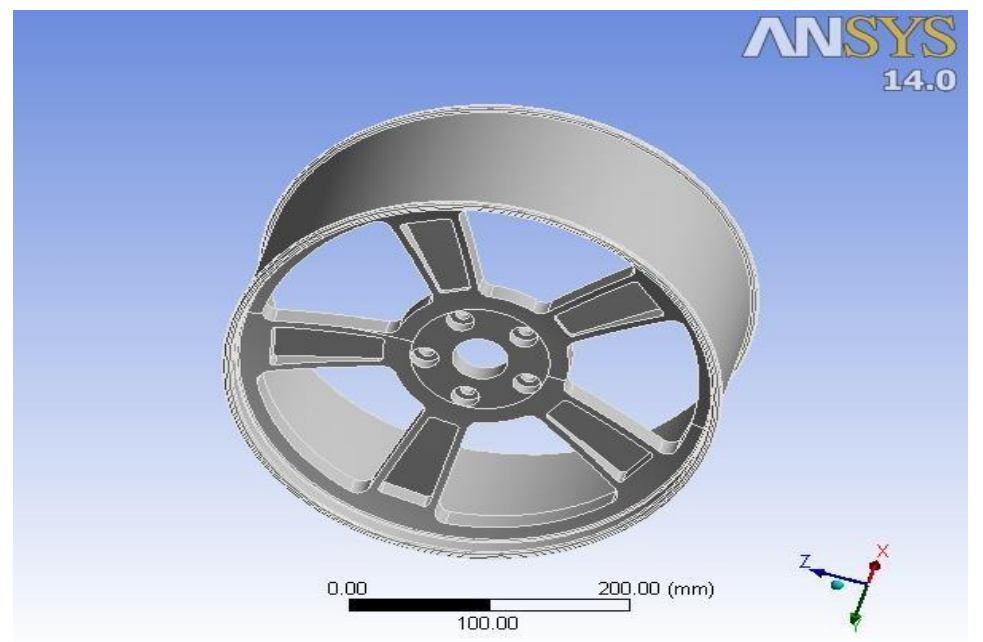

Figure 1: Model of Wheel Rim.

In the case or radial test 10 -noded tetrahedral solid element is used

\section{DESIGN AND ANALYSIS OF EXISTING MODELIN ANSYS}

Alloy wheels are automobile wheels which are made from an alloy of aluminium or magnesium metals or sometimes a mixture of both. Alloy wheels differ from normal steel wheels because of their lighter weight, which improves the steering and the speed of the car. Alloy wheels will reduce the unstrung weight of a vehicle compared to one fitted with standard steel wheels. The benefit of reduced unstrung weight is more precise steering as well as nominal reduction in fuel consumption. Alloy is an excellent conductor of heat, improving heat dissipation from the brakes, reducing the risk of brake failure under demanding this collecting data from reverse engineering Process from existing model.

\section{Meshing}

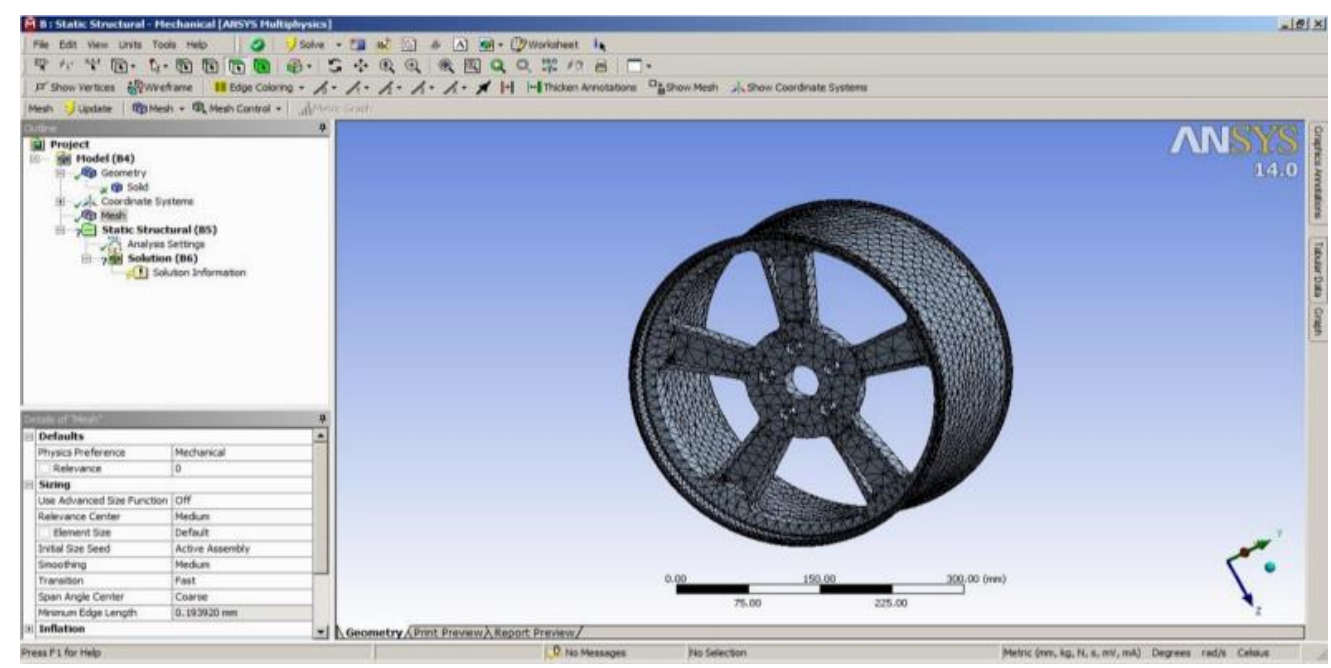

Figure 2: Meshing of Wheel Rim. 
Total Deformation of Wheel Rim

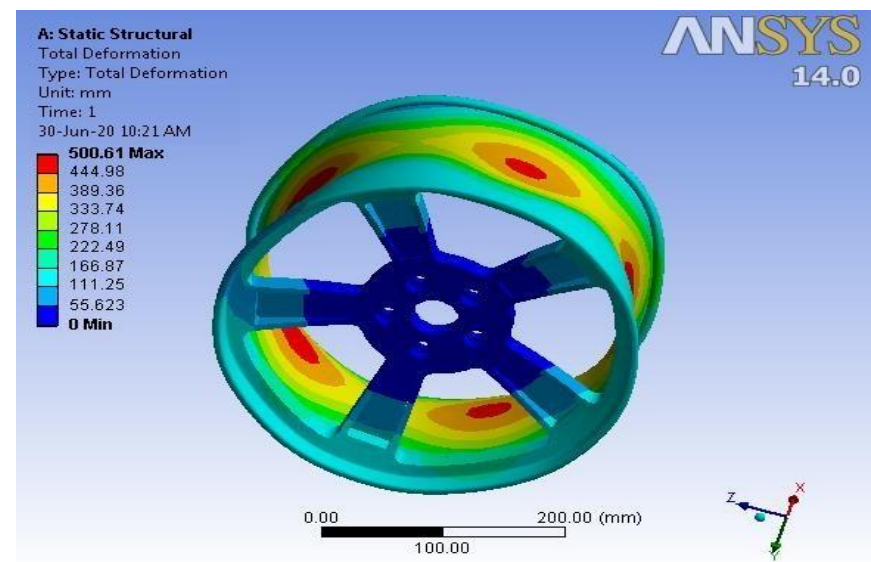

Figure 3: Total Deformation of Wheel Rim.

Equivalent Elastic Strain

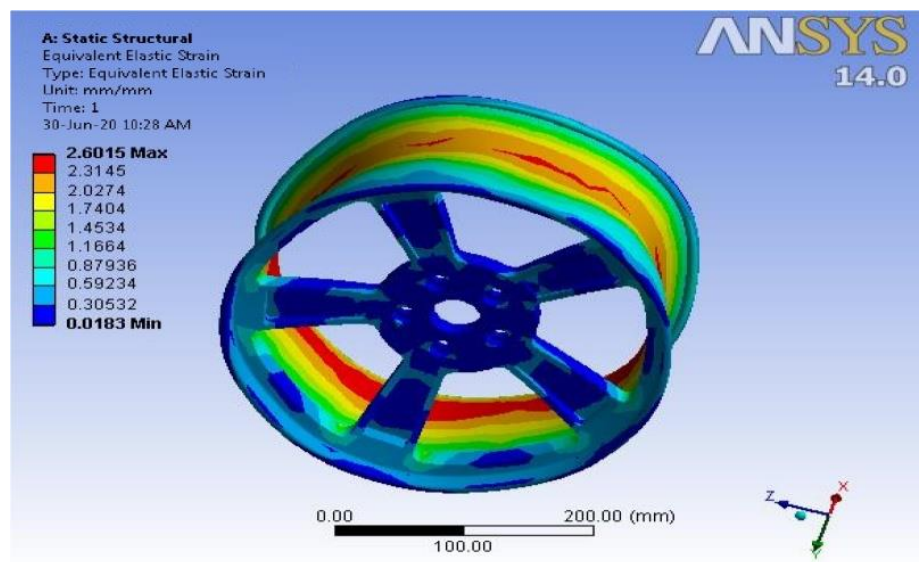

Figure 4: Equivalent Elastic Strain of Wheel Rim.

\section{Equivalent Stress}

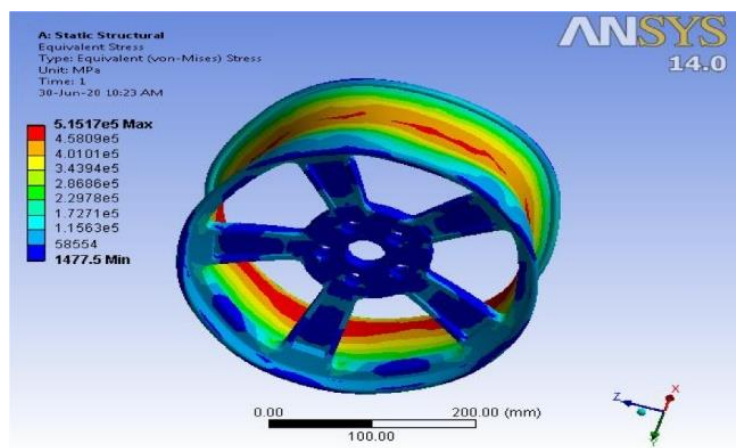

Figure 5: Equivalent Stress in Wheel Rim.

\section{PROPOSED MODEL DESIGN AND ANALYSIS}

At present four wheeler wheels are made of Aluminium Alloys. In Design change proposal in order to reduce weight of wheel in this section, design change proposals will be given in order to reduce wheel weight and these proposals will be investigated for durability and NVH considerations. In order to avoid undesired interior noise the natural frequency of the 
wheel should be higher than $350 \mathrm{~Hz}$ since this value is considered as critical frequency. Moreover, durability availability will be checked on defined condition in SAE J328. $5 \mathrm{~mm}$ metal removing has been applied by to the areas that black colour and marked with in the oval shape. Metal removing has been applied in order to reduce weight.

\section{Material Used}

Aluminium Alloy A 356.2

\section{Mechanical Properties}

- Ultimate strength

- Density

- Yield strength

\section{Composition}

$\begin{array}{lc}\text { Silicon } & 6.5-7.5 \% \\ \text { Iron } & 0.12 \% \\ \text { Manganese } & 0.05 \% \\ \text { Magnesium } & 0.3-0.45 \% \\ \text { Zinc } & 0.50 \% \\ \text { Titanium } & 0.20 \% \\ \text { Copper } & 0.10 \% \text { Others } \\ \text { 0.15\% (Remaining Aluminium) } & \end{array}$

\section{Experimental Work}

Experimental Setup for Alloy Wheel to know resonance natural frequency special purpose machine was used for Electrodynamics Vibration Shaker. The experimentation for alloy wheel is carried out using an Electrodynamics Vibration Shaker. The wheel is held in position with other operating conditions identical to the application. Test Conditions are Resonance Search from $50 \mathrm{~Hz}$ to $750 \mathrm{~Hz}$, Acceleration 1g. Device under test mounted on 4 holes to the shaker table. Control accelerometer mounted with adhesive on shaker table. Monitor accelerometer mounted on device under the test with adhesive. Controlling and monitoring is done with vibration controller.

\section{Analysis}

Designers and engineers primarily use structural simulation to determine the strength and stiffness of a product by reporting component stress and deformations. The type of structural analysis performs depends on the product being tested, the nature of the loads, and the expected failure mode: A short/stocky structure will most likely fail due to material failure (that is, the yield stress is exceeded). For the given below specification of the allow wheel, the static analysis is performed using solid works to find the maximum safe stress and the corresponding pay load. After geometric modeling of the alloy wheel with given specifications it is subjected to analysis. 
The Analysis involves the following discretization called meshing, boundary conditions and loading. About analysis with Solid Works: Solid Works Simulation Xpress is design analysis software that is fully integrated in solid Works. SolidWorks Simulation Xpress simulates the testing of your part's prototype in its working environment. It can help you answer questions like: how safe, efficient, and economical is your design. Solid Works Simulation Xpress is used by students, designers, analysts, engineers, and other professionals to produce safe, efficient, and economical designs.

Fatigue Analysis: During design validation, a structure is exposed to both static strength tests and fatigue tests. However, once a structure is deployed, it spends the vast majority of its lifetime being subjected to smaller repeated forces that can cause cumulative damage over time. For this reason, testing the durability of a structure makes up a larger proportion of the tests that are run. Durability is one of the most important attributes that structures can possess. Fatigue testing measures durability and is defined as the repeated mechanical loading of a structure to determine failure points. It requires complex analysis using the field of fracture mechanics, which is the analysis of material flaws to discover those that are safe and those that are liable to propagate as cracks and cause failure. Add an event as constant amplitude for a required number of cycles, with zero based condition. Then define S-N curve for the applied material, as log-log graph. The Stress-Life (S-N) or Total Fatigue Life method is widely used for HCF applications. During HCF testing, a material spends the majority of life in a state where the cracks are very small, the growth is controlled, and the structure integrity is retained. As noted earlier, the applied stress stays within the elastic range of the material. Total Life is determined by running multiple specimen tests at a number of different stresses. The objective is to identify the highest stress that produces a fatigue life beyond 10 million cycles. This stress is also known as the material's endurance limit.

\section{Meshing}

Meshing involves division of the entire of model into small pieces called elements. This is done by meshing. It is convenient to select the Standard mesh because of wheel structures, so that shape of the object will not alter.

\section{Boundary Conditions}

To ensure the accuracy and reliability of the analysis result, the structural and mechanical model of the rear wheel is established. Shows the existing models 14 and redesigned model Net weight of the motorcycle is $163 \mathrm{~kg}$ and the maximum allowable Load 6. The tyre used is a common version with inner tube filled to gas pressure $0.28 \mathrm{Mpa}$, uniformly distributed on the exterior ring surface of wheel. To ensure reliability of the analysis, the sum of motorcycle net weight and maximum allowable load was applied to the rear wheel alone. The sum was considered to be the maximum load, which was distributed on the rim surface. By considering, the maximum load is equal to motorcycle weight including rider and all loads. 


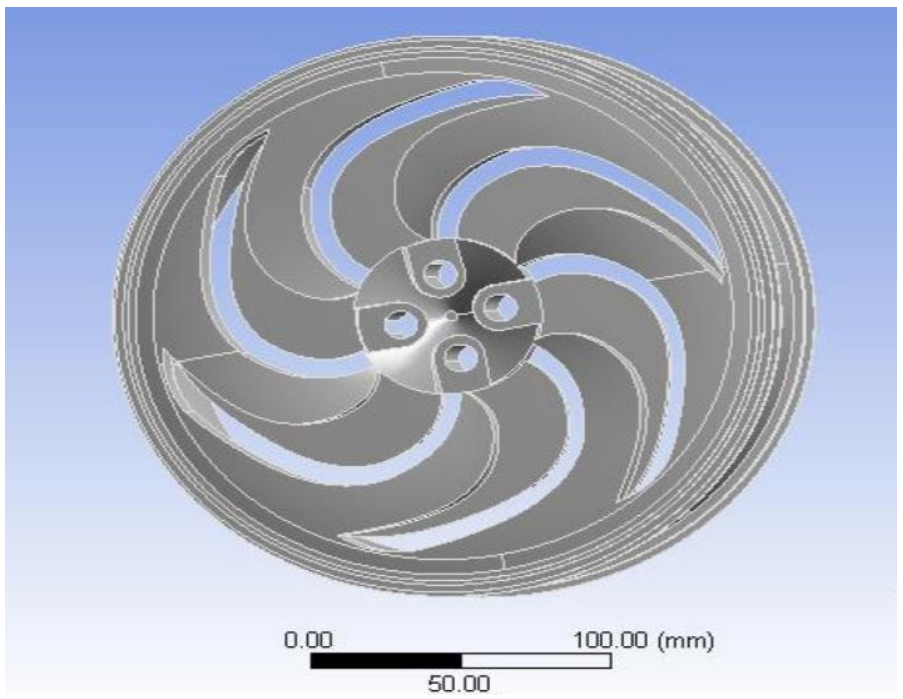

Figure 6: Proposed Model.

\section{Applying Boundary Condition}

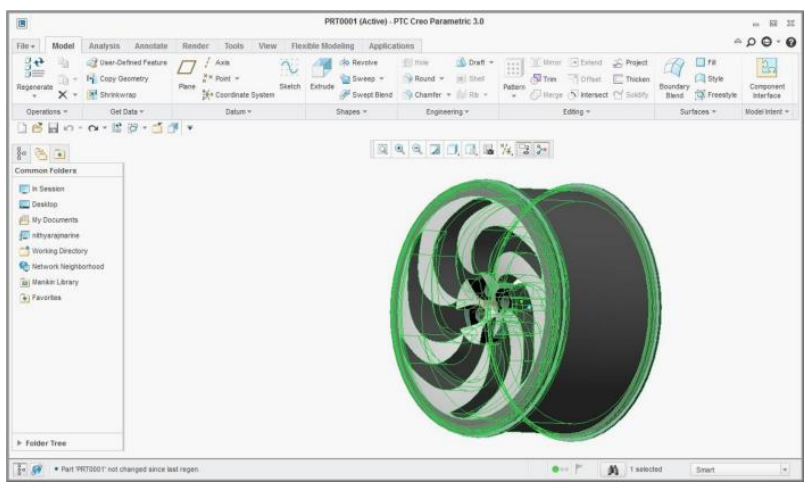

Figure 7: Applying Boundary Condition to Proposed Model.

\section{Total Deformation}

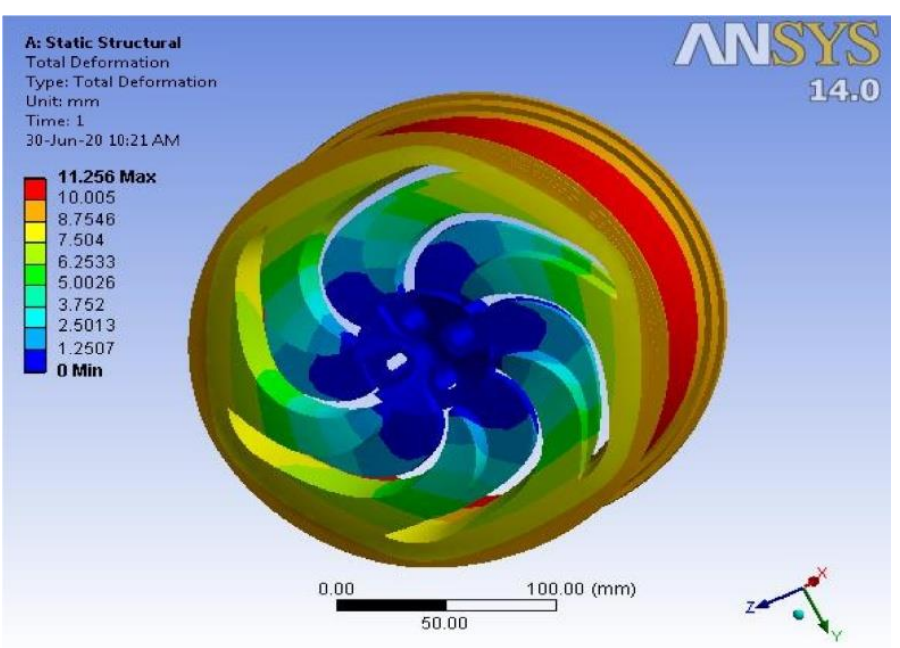

Figure 8: Total Deformation of Proposed Model. 


\section{LOAD CASE EXPLANATION}

\section{Load Case Bending}

In the case of bending test a vertical load of $2755.16 \mathrm{~N}$ is applied at a distance of $1 \mathrm{~m}$ from the center of the hub. Before applying the load the model should be meshed property. There are six degrees of freedom of which there are three translations and three rotations. The type of constraints depends upon the type of model. In our case we have arrested all the six degrees of freedom.

In case of wheel outer rim is constrained for all six degrees of freedom. The outer rim is selected by means of a selection set and displacement is defined by means of node by obeying the conditions as mentioned above. After the constrained are made by using appropriate commands it can be screened by using the command DPLOT. The load is applied to the node in Y direction (downward direction) after the constraints are specified for the model. The forces are plotted on the screen using the command FPLOT.

Before running the analysis the model should be feed with sufficient data such as material property, real constants and element group. Under material property, the property such as density, Poisson's ratio, Young's modulus should be given as data for material, which have been selected for the model. Aluminum alloy (A 356.2) is selected as material for our model with density $2700 \mathrm{~kg} / \mathrm{m}^{\wedge} 3$, Poisson's ratio 0.3 and Young's modulus of $67500 \mathrm{~kg} / \mathrm{m}^{\wedge} 2$. Under element group it is necessary to check the attributes of elements mentioned in the model and then the data's are checked using DATA CHECK command. To verify that all needed attributes for an element are being defined, RUN CHECK does more elaborate checking including element connectivity. With the use of analysis option, the desired analysis (RUN STATIC ANALYSIS) is made to run for the model and the necessary results are plotted.

\section{Load Case Pressure}

In case of pressure loading, the pressure of $3.5 \mathrm{~kg} / \mathrm{cm} 2$ is applied through the circumference of the wheel. Before applying the load the model should be constrained properly. The type of constrain depends upon the type of model. In our case we have constrained all the six degrees of freedom.

In the case of pressure loading, bolts are constrained for all six degrees of freedom. The bolt is selected by means of a selection set and displacement is defined by means of nodes by obeying the conditions as mentioned above. After the constrained are made by using appropriate commands it can be screened by using the command DPLOT. The load is applied to the node in Y direction (downward direction) after the constraints are specified for the model. The forces are plotted on the screen using the command FPLOT. Before running the analysis the model should be feed with sufficient data such as material property, the property such as Density,

Poisson's ratio, Young's modulus should be given as data for material, which have been selected for the model. Aluminum alloy (A 356.2) is selected as a material for our model with density $2.7 \mathrm{E}-6 \mathrm{~kg} / \mathrm{mm} 3$, Poisson's ratio 0.3 and young's modulus of $0.675 \mathrm{E} 5 \mathrm{~kg} / \mathrm{mm} 2$

\section{Load Case Centrifugal}

In the case of centrifugal loading, the wheel is constrained at the bolts and is constrained for all 6 degrees of freedom. In the case of centrifugal loading the wheel has to be transferred to the global axis i.e., keeping the axis at the centre of the 
wheel assuming it to be revolved with reference to that axis. Cosmos has got a special feature of calculating the centrifugal load if we specify the angular velocity.

The element group and material properties are defined appropriately and the analysis is made to run. The normal stress is plotted along Y- axis and is shows very negligible amount of stress coming on to the wheel.

\section{Load Case Vertical}

In case of vertical loading, a vertical load of $13998.375 \mathrm{~N}$ is applied vertically from downwards. Before applying the load the model should be constrained properly. The type of constrain depends upon the type of model. In our case we have constrained all the six degrees of freedom.

In the case of Vertical loading, bolts are constrained for all six degrees of freedom. The bolt is selected by means of a selection set and the displacement is defined by means of nodes by obeying the conditions as mentioned above. After the command DPLOT, the load is applied to the node in Y direction (downward direction) after the constraints are specified for the model.

After plotting the nodes a temporary point is created at the centre of the hub. This point is extruded in such a way that it passes on to 60 degree on both sides from the centre of the wheel. The nodes are selected on either side of the wheel in the selection set and the load is applied only to that portion where the tyre is getting seated. The forces are plotted on the screen using the command FPLOT before property, real constants and element group. Under material property, the property such as Density, Poisson's ratio, Young's modulus should be given as data for material, which have been selected for the model. Aluminium alloy (A 356.2) is selected as a material for our model with density 2.7E-6 kg/mm2, Poisson's ratio 0.3 and Young's modulus of $0.675 \mathrm{E} 5 \mathrm{~kg} / \mathrm{mm} 2$.

Under element group it is necessary to check the attributes of elements mentioned in the model and then the data's are checked using DATA CHECK command. To verify that all needed attributes for an element are being defined, RUN CHECK does more elaborate checking including element connectivity. With the use of analysis option, the desired analysis (RUN STATIC ANALYAIS) is made to run for the model and necessary results are plotted.

\section{Von Mises Stress Distribution}

When a machine member is subjected to Bi-axial or tri-axial stress system, the determination of failure stress is more complicated. Hence some theories are found out for finding the failure properties of different materials. Among these theories, the von Mises theory or maximum distortion energy theory is calculated theoretically and verified with practical results.

Von Mises Theory:

$\mathrm{Vm}^{2}=\sigma 1^{2}+\sigma 2^{2}+\sigma 3^{2}-\sigma 1 \sigma 2-\sigma 2 \sigma 3-\sigma 1 \sigma 3$

\section{CONCLUSIONS}

The wheel is analysed for the following two load conditions:

- Bending endurance test.

- Radial endurance test. 
In radial endurance test three conditions are checked.

- $\quad$ Pressure Loading

- Centrifugal Loading

- $\quad$ Vertical Loading.

The wheel is constrained appropriately and the loads are calculated based on the specifications and applied to appropriate nodes. The wheel is analysed for the calculated loading condition and the stress plot is obtained. In the case of bending test normal stress along Y-axis shows compression on the top rib and tension on the bottom rib and compression on the bottom rib.

In the case of pressure loading, normal stress along $\mathrm{X}$-axis shows compression on the top rim and on the inside portion of the rim there is a gradual transition from compression to tension. Normal stress along $\mathrm{Y}$-axis shows bending stress coming on to ribs because when the rim is getting compressed, it forces the rib to move outwards. In the case of vertical loading normal stress along Y-axis shows tension on the outer rib and compression on the outer side of the rib. When a section plot is taken it will show a gradual transition from tension to compression.

The file, which is created in ANSYS, is equivalent with the provision for flexibility by which stress can be modified to suit any possible situation that may arise in future. The linear static stress analysis is performed for the present wheel rim. The future work involves different types of analysis such as Impact testing, dynamic and vibration analysis

\section{REFERENCES}

1. R.B.Gupta, Automobile Engineering, Tech India Publication Series. PSG Design Data Book Published by DPV Printers.

2. Warren dale, PA Formulae SAE Rules, SAE International Introduction to mechanical engineers, Ed Automobiles wheels and tyres, E conference publications.

3. Krishnaveni, J., G. Sowmya, and U. Sudhakar. "Thermal Analysis of Cylinder Head by Using Finite Element Analysis." International Journal of Mechanical Engineering (IJME) ISSN (P), 23192240 (2014).

4. Reddy, J.N., An introduction to the finite element method McGraw Hill PublishingCompany

5. Benziger, B., P. Anu Nair, and P. Balakrishnan. "Review paper on thermoelectric airconditioner using peltier modules." IJME 4.

6. Cook, R.D., Finite element modelling for stress analysis, John Wiley \& Sons, Moaveni, S., Finite Element Analysis, Theory and application with ANSYS, Prentice Hall, Pág: 1-36

7. S. Vikranth Deepak, Modeling and analysis of alloy wheel for four wheeler vehicle, Research Paper.

8. Patel, MEHUL D., et al. "Adaptive physiological and biochemical responses of dairy animals to heat stress: a review." International Journal of Applied and Natural Sciences 5.1 (2016): 107-116.

9. WU Li-hong1, LONG Si-yuan2, and GUAN Shao-kang: Replacement of A365 with AM60A, service stress distribution in the wheel becomes more uniform

10. Baba, M., C. Tumba, and A. Issa. "Formulation and Performance Assessment of Fixed Oils Based Cutting Fluids in Machining Operation." Int. J. Mech. Eng 7.6 (2018): 11-22. 